\title{
Relatos de los equipos de salud mental de atención primaria (aps) acerca del abordaje de la violencia de género en la pareja
}

\author{
Discourses of primary mental healthcare teams \\ on addressing gender violence within the context of relationships
}

${ }^{1}$ Depto. Salud Pública, Facultad de Medicina, Universidad de Valparaíso. Errázuriz 1834. Valparaíso Quinta Región de

Valparaíso Chile.

beatriz.salgado@uv.cl

\begin{abstract}
Violence against women within relationships is a problem which is in the public eye and is mainly associated with the gender factor. It is therefore crucial to incorporate a gender perspective in order to understand and intervene in this phenomenon. A qualitative study was conducted using semi-structured interviews and applying content analysis, to analyze if the declared discourse of the Primary Mental Healthcare teams in Valparaíso (physicians, psychologists, social workers), regarding tackling violence against women within the context of a relationship includes perceptions, knowledge and ideas based on the gender perspective. There is a general consensus on how to deal with battered women, as for all of them it is very important to create an empath$i c$, therapeutic space of containment. Multidisciplinary and intersectoral work is considered important in the work discussions and the risk of repeat victimization was mentioned. In general, there was no intention to empower women from a gender perspective, with the exception of some psychologists (males and females). This work could lead to a critical evaluation of the various discourses of Primary Mental Healthcare Teams in addressing gender-based violence.
\end{abstract}

Key words Gender violence, Domestic violence, Partner violence, Primary healthcare, Mental health
Resumen La violencia contra las mujeres en el contexto de la relación de pareja es un problema situado en la agenda pública, asociado a la determinación de género. Incorporar la perspectiva de género es, por tanto, crucial para poder entender e intervenir en este fenómeno. Para analizar si los discursos que poseen los profesionales de los Equipos de Salud Mental de APS de la Comuna de Valparaíso (Médicos/as, Psicólogos/as, Asistentes Sociales) respecto del abordaje que realizan sobre violencia doméstica presentan saberes, ideas o nociones basados en perspectiva de género, se realizó estudio con metodología cualitativa, a través de entrevistas semiestructuradas y se realizó un análisis de contenido. Hay consenso sobre la forma de abordar a las mujeres agredidas, para todos es muy relevante generar un espacio terapéutico empático, de contención. Se valora en los discursos el trabajo multidiciplinario e intersectorial. Se mencionan prácticas de riesgo de generar revictimización. No existe intencionalidad hacia un empoderamiento desde una perspectiva de género, salvo en el caso de algunos/as psicólogos/as. El presente trabajo podría aportar a la evaluación crítica de los distintos discursos de los Equipos de Salud Mental de APS en el abordaje de la violencia de género.

Palabras Clave Violencia de género, Violencia doméstica, Violencia de pareja, Atención Primaria, Salud Mental 


\section{Introducción}

La violencia contra las mujeres en el contexto de la relación de pareja es un problema situado en la agenda pública. Se asocia principalmente, pero no estrictamente, al género. Es considerada un tipo de violencia que pueden sufrir las mujeres a causa de pertenencia al género femenino ${ }^{1-4}$. Incorporar la perspectiva de género, desde un enfoque de determinantes sociales de la salud, es crucial para entender e intervenir este fenómeno.

Para analizar el maltrato y abuso sobre las mujeres en las relaciones de pareja se utilizará la perspectiva de poder y control implícita en la teoría de género ${ }^{5,6}$. Entendemos que este tipo de violencia pasa a ser uno más de los elementos de control y poder que facilita y mantiene la identidad del género masculino en oposición al femenino y acceso diferencial al poder. Poder que perpetúa los privilegios que la masculinidad hegemónica posee dentro de una cultura fundamentada en la desigualdad entre los géneros ${ }^{7}$.

Es por ello que se utilizará la siguiente definición del concepto de violencia de género:

Una acción de potencia y de fuerza que se ejerce sobre hombres y mujeres basada en las construcciones sociales y cultural, y en razón de la pertenencia a un sexo específico o en razón de su orientación sexual. Dicha acción se manifiesta de acuerdo con la posición y condición de subordinación en la que se encuentra cada sujeto en las diferentes dinámicas del poder. Estas acciones se hacen evidentes en las relaciones interpersonales y sociales, que operan cotidianamente para el funcionamiento y desarrollo de las instituciones ${ }^{8}$.

Dado que la mayoría de las mujeres establecen contacto con sistemas de salud en algún momento de sus vidas (control de embarazo y parto, control de la fertilidad, en busca de atención para sus hijos y otros familiares), los centros de APS y urgencia son sitios importantes para detectar mujeres maltratadas, brindarles apoyo y enviarlas, si fuera necesario, a servicios especializados. Es relevante destacar las dificultades del sector salud al trabajar este tema. Existen diversos estudios que muestran la renuencia del sistema sanitario a considerar la Violencia Doméstica, aunque haya sido definida mundialmente como problema de salud pública ${ }^{1,9-13}$.

Estudios muestran lo poco familiarizados que se encuentran los profesionales del área con la violencia conyugal y lo difícil que resulta asumir estos casos. Un estudio realizado en centros de APS en Seattle, EEUU detectó que el 50\% de los médicos y el 70\% de las enfermeras/paramédicos creían que la prevalencia de la violencia doméstica en su centro de salud era rara ó muy rara (menos de 1\%). Además el 45\% de los médicos nunca o casi nunca preguntaban acerca de violencia doméstica al tratar pacientes de alto riesgo de sufrirla ${ }^{9,11}$.

Lo mismo se observa en los centros de urgencia, donde existen bajas tasas de diagnósticos de violencia de pareja, incorporando elementos recordatorios de violencia de género (como instrumentos diagnósticos), la tasa aumenta considerablemente ${ }^{12,13}$. Algo muy similar se muestra en la consulta psiquiátrica, evidenciándose la poca actitud de pesquisa que tienen los profesionales de la salud en general. Al evaluar si el sexo del terapeuta (Médicos/as de Familia) hace alguna diferencia en las percepciones y actitudes que tienen frente al tema de la violencia de pareja, dos estudios encontraron que las Médicas se mostraban más empáticas, comprometidas emocionalmente, comprensivas y abiertas a tratar casos de violencia conyugal en comparación a los varones ${ }^{11,14}$.

Las características propias del fenómeno de la Violencia Doméstica generan dos conductas que se potencian mutuamente: por un lado, la mujer tiende a ocultar su condición de víctima de violencia, por motivos desde la vergüenza al miedo; por otro, los médicos y otros profesionales no detectan ni identifican el factor violencia doméstica en el contexto de una consulta clínica o psiquiátrica. En palabras de Corsi:

La actitud de los profesionales del área Salud que reciben las consultas es decisiva: cuando consideran (a partir de un conocimiento específico del tema) el problema de la violencia y el maltrato como posible factor generador de sintomatología, dan el primer paso para una adecuada respuesta a la mujer que consulta. En cambio, si lo desconoce, lo niega o lo minimiza, está sentando las bases para una segunda victimización a la cual es sometida la mujer ${ }^{15}$.

Archer ${ }^{10}$ plantea que los Médicos de Familia, desde la posición privilegiada que poseen (el rol que juegan en la APS y el prestigio médico en la sociedad) pueden afectar de manera muy importante en numerosos problemas de salud que presentan particularmente las mujeres, se dirige a las actitudes que deberían tener y las formas de actuar para empoderar, desde una perspectiva de género, a las mujeres que sufren violencia, lo que incidiría positivamente en eliminarla.

Actitudes como: evitar la negación del problema, es decir reconocer que la violencia es muy habitual y que una gran cantidad de mujeres conviven con ella; respetar los silencios de las muje- 
res, es decir su derecho a negar u ocultar su situación de violencia de tal manera de evitar la revictimización; no culpar a la víctima, considerar que bajo ningún punto de vista y ninguna circunstancia el abuso o acoso sexual, la violación o el trato abusivo hacia las mujeres es aceptable o defendible; buscar el empoderamiento de las usuarias por ejemplo, a través de establecer una relación médico-paciente más horizontal; conocer las redes disponibles para el apoyo a las mujeres en situación de violencia y mantenerse en continua actualización en estos temas, como se hace en otros temas de salud ${ }^{10,16}$.

Trabajar con mujeres desde una perspectiva de género implica ayudarlas a conectar aquello que les ocurre con la socialización de género dirigida hacia las mujeres, lo que les dificulta a ellas identificarse y tomar decisiones vitales, elecciones que pueden ser contrarias a lo que otros sugieren. Trabajar con mujeres de una manera empoderadora, respecto a la capacidad de hacer elecciones por sí mismas, poder generar soluciones para sus problemas, independiente de que esas decisiones sean tomadas de forma inmediata o a largo plazo, parecen ser actitudes que van a permitir al menos que las mujeres agredidas perciban una mirada distinta de la situación de violencia en la que viven ${ }^{10}$.

Sin duda es posible pensar que otros profesionales que trabajan con mujeres violentadas, más allá de los distintos roles y abordajes terapéuticos que puedan tener, pueden contribuir al empoderamiento de éstas, incorporando este tipo de actitudes y acciones a su quehacer.

El feminismo ha realizado importantes críticas a las formas de intervención terapéuticas tradicionales en Salud Mental, ya que tales terapias tienden a infantilizar y desempoderar a las mujeres, facilitan la culpabilización, por lo que se plantea que las intervenciones terapéuticas en mujeres maltratadas no podrían ser realizadas por cualquiera, básicamente por los sesgos de género:

Los psicoterapeutas, con frecuencia inadvertidamente, han aportado a la pérdida de autoestima de la mujer al unirse en la conspiración de silencio que existe alrededor de los incidentes de maltrato y por concentrarse en la naturaleza "provocativa" de las mujeres cuando esos incidentes son revelados en sesiones de terapia ${ }^{17}$.

Las intervenciones más exitosas en mujeres agredidas son los grupos de autoayuda, la intervención en crisis, la psicoterapia individual y en terapia de grupo y, solamente en un número muy limitado de casos se podría realizar terapia de pareja o de familia. Algunos autores/as señalan que es imposible realizar terapia de pareja en estos casos, otros/as que sólo se podría realizar en casos puntuales, dado el extremo desequilibrio de poder en la pareja. Esto nos lleva a pensar que el espacio terapéutico que se genera en APS no debiese ser utilizado para este tipo de intervenciones. En el caso de los agresores, lo que se ha recomendado es tratarlos por separado en grupos de maltratadores ${ }^{17,18}$.

En general las estrategias de intervención existentes, a nivel mundial, se han concentrado en sensibilizar a los prestadores de asistencia sanitaria, alentar la detección sistemática del maltrato y establecer protocolos para el tratamiento adecuado. Una cantidad cada vez mayor de países han iniciado proyectos para capacitar a los trabajadores de la salud para que puedan identificar y dar respuesta al maltrato. Varios países latinoamericanos también han incorporado normas para abordar la violencia doméstica en las políticas del sector salud ${ }^{1}$.

En Chile, el Ministerio de Salud (MINSAL) considera a la violencia intrafamiliar como prioridad programática. Las primeras acciones derivadas desde la autoridad sanitaria se orientaron a realizar proyectos pilotos para el diagnóstico y sensibilización de los equipos de salud y a las usuarias del sistema. Dentro de este proceso se llegó a determinar que, si bien la violencia intrafamiliar (incluida la violencia hacia menores, adultos mayores y mujeres) genera un gran impacto emocional en los equipos de salud, esto no se traduce en acciones concretas y efectivas, tanto por la falta de tiempo, la carga asistencial y especialmente por la carencia de capacitación sobre el tema ${ }^{19}$.

El MINSAL ha elaborado, 4 documentos relativos al tema de Violencia Intrafamiliar destinados a la APS, que contienen directrices para el abordaje del problema ${ }^{20-23}$. Según el informe del Observatorio de Equidad de Género en Salud de 2005, en Chile, no existían orientaciones para indagar violencia intrafamiliar en las normativas de registro de la atención ${ }^{24}$.

La introducción de cambios en los procedimientos asistenciales (tales como estampar un recordatorio para el personal de salud en la ficha de la paciente o incorporar preguntas sobre el maltrato en los formularios de ingreso, introducir instrumentos de tamizaje en la práctica clínica, etc.) surte efecto sobre el comportamiento del personal sanitario, aunque también es importante hacer frente a creencias y actitudes muy arraigadas $^{1}$.

Si bien la indagación activa de maltrato (preguntar sobre posibles antecedentes de agresión 
por parte de la pareja) se considera una práctica adecuada, ya que las mujeres aceptan de buen grado que se les pregunte sobre la violencia desde una posición moralmente neutra ${ }^{16}$, no se ha evaluado de manera sistemática si la detección del abuso puede mejorar la seguridad de las mujeres o su comportamiento de búsqueda de ayuda y en qué condiciones. Es importante tener en cuenta que el riesgo de femicidio aumenta 6 veces cuando la mujer se separa del agresor ${ }^{1}$.

\section{Objetivo}

Analizar si los discursos que poseen los profesionales de los Equipos de Salud Mental de APS de la Comuna de Valparaíso respecto del abordaje que realizan sobre violencia de género en la pareja presentan saberes, ideas o nociones basadas en perspectiva de género.

\section{Metodología}

Estudio cualitativo exploratorio, como método de producción de información se utilizó la entrevista semi-estructurada para obtener información a nivel profundo y acceder, a través de un portavoz, a representaciones de grupos determinados, con identidades de existencia construidas en base a condiciones históricas, socio-económicas y culturales específicas, que permiten la reproducción de sistemas de posturas, opiniones y prácticas semejantes ${ }^{25}$.

El sujeto de estudio está constituido por los profesionales que participan en la atención de pacientes del Programa de Salud Mental (Psicólogas/os, Médicos/as y Trabajadores/as Sociales) de la red de APS de la Comuna de Valparaíso (14 Centros), es decir que pertenecen a los equipos de salud mental de tales establecimientos, con al menos un año de experiencia trabajando en esas áreas dentro del Centro de Salud, respectivo. El programa de Salud Mental incluye la atención de problemas de salud relacionados con la Violencia Intrafamiliar, existiendo un subprograma para usuarias víctimas de violencia de pareja ${ }^{20-23}$.

Los Criterios de segmentación fueron: profesión y sexo de los profesionales. Se realizaron 11 entrevistas con las cuales la investigadora consideró lograda la saturación de la información, entre estas, dos Psicólogos, dos Psicólogas, dos Médicas, dos Médicos y tres Asistentes Sociales (no había trabajadores sociales varones en el grupo estudiado).
Como técnica de sistematización de la información se utilizó el análisis de contenido ${ }^{25}$ de los relatos. Fundamentándose en el marco del constructivismo social ${ }^{26}$, desde un enfoque feminista de la teoría de género ${ }^{6}$, en base al objetivo de la investigación y a partir de los textos recabados en las entrevistas, se identificaron códigos y se definieron las principales categorías de análisis, a partir de las cuales se desprendieron subcategorías y se organizó la interpretación del contenido de los relatos.

\section{Resultados}

Globalmente, en todos los perfiles aparece el concepto de que para el abordaje de las usuarias que presentan situaciones de violencia en su relación de pareja es imprescindible la actitud de escucha. También se perciben como valorables aspectos como la empatía, la calidez en la atención, el establecer una buena relación terapeuta/paciente para lograr una relación de ayuda, la cual es vista como fundamental tanto para pesquisar como para realizar un buen seguimiento de estos casos.

Lo principal es la acogida, acogerla con empatía, generar un espacio donde ella se pueda expresar, donde se sienta que está siendo escuchada más que juzgada y que pueda sentir la confianza para contar y entregar todos los elementos para poder evaluar el riesgo, hacer el espacio y hacer más natural la situación que por muy critica que te parezca darle toda la naturalidad para que ella te pueda expresar todo lo que siente, y también describir todas las situaciones, creo que también es importante entregarle un poco de calma, de tratar de contenerla y hacerla sentir que tiene un apoyo, o sea, que no está sola y que alguna solución tú le vas a poder ayudar a encontrar a su problema. (AS, E9, L149)

Se valora la importancia de la contención en el primer momento, evitando el referirse a su situación de violencia de forma tan directa, en un principio.

trato de irme al tiro a contenerla, protegerla y en el fondo que... es súper importante, que en realidad les digo a todos los pacientes que estos son 4 paredes y que de aquí no va a salir, o sea... y que de aquí en adelante sonó y no se va a librar de nosotros, o sea que la vamos a ayudar, la vamos a proteger y que ante cualquier cosa venga a cualquier hora. (PsM, E6, L121)

Luego de unas tres sesiones yo empiezo a mostrarles pero, ¿usted se ha dado cuenta de esto?, mire que está pasando con su marido... entonces em- 
piezo a mostrarle ahí, algunas han enganchado $y$ ha resultado aquí también algunas, pero hay que hacer un trabajo, elaborar un setting crear un vinculo con el paciente. (PsM, E5, L58)

Sin embargo, algunos/as entrevistados/as perciben que la falta de tiempo de atención en la APS les dificulta la posibilidad de abordar adecuadamente estas complejas situaciones de consulta.

entonces tú si te das un tiempito, a pesar que acá el tiempo casi no te da, y comienzas a preguntar cómo están emocionalmente, la relación... entonces empieza a salir a flote, tienes que sacar con cucharita y ahí sale. (MM, E2, L20)

Cuando uno ya anda oliendo o sospechando algún hecho concreto de violencia, uno lamentablemente tiene que sentarse bien en el sillón y asumir que va a perder un montón de tiempo hablando con la paciente. (MH, E1, L119)

Dentro de este mismo contexto surge la idea de que no es conveniente enjuiciar, culpabilizar, ni ser directivo/a en las intervenciones.

Eso. Tener el tiempo y escucharla, sobretodo y no darle una orden precisa o consejo tan directo de que tiene que hacer ella, eso se dejará para una segunda etapa.... Porque... si le digo, le hago ver lo objetivo que está viviendo ella, lo mal que ella estuvo, tal vez... Soportando en esta relación de violencia, puedo generar en ella también una reacción de rechazo, así como un reto. Así que primero logro mantener una confianza sobre todo con harto tiempo, de que ella realmente me vea a mí como alguien que la está apoyando, más que alguien que la está dirigiendo. (MM, E1, L137)

porque a veces uno tratando de meter la $\mathrm{cu}$ chara sin querer puede juzgar, puede criticar, no teniendo los conocimientos. (PsM, E6, L163)

Igualmente, en todos los perfiles, se reconoce la importancia de tener una actitud de sospecha para poder pesquisar los casos frente a consultas de otras causas lo cual es visualizado como algo que se debe realizar con tiempo y sin presionar a la consultante.

va a ser recurrente en la medida en que nosotros también agudicemos nuestro oído, hay muchas señales que las personas nos dan y que muchas veces no tomamos. ( $\mathrm{PsH}, \mathrm{E} 7, \mathrm{~L} 175)$

Entonces indagando y conversando con ella de poco a poco va saliendo. (MM, E2, L133)

En el caso del perfil de médicos y de médicas, se considera a la pesquisa como una de sus funciones principales dentro del manejo integral de los casos de violencia.

Así que la táctica que manejamos aquí siempre que se desliza algún tema de violencia es prepararnos a perder tiempo, o sea, a perder el tiempo... a usar el tiempo en ella. No apurarse... Nosotros los médicos, en general, nos dedicamos más que nada a la pesquisa. (MH, E1, L127)

En la forma más activa, [de la forma de participación del entrevistado en los casos de violencia] porque donde primero viene el paciente es a la consulta o al médico, entonces te plantea el problema de la violencia enmascarado. (MH, E3, L20)

En todos los perfiles se percibe como importante la búsqueda de redes para las mujeres que consultan por violencia en su relación de pareja, redes tanto institucionales como comunitarias, familiares, etc.

que la paciente se dé cuenta que uno está dispuesto a ayudarla, dispuesto a que busque redes de apoyo, no solamente redes de salud, si no que redes sociales. (MH, E3, L138)

como ver la red que tiene de apoyo, y en la medida de eso... (PsM, E5, L57)

Para perfiles de médicos, médicas y asistentes sociales, es importante el abordaje multidisciplinario que se realiza, el cual es mencionado más explícitamente por ellos/as, asociado a la referencia a un abordaje integral de la familia de la consultante.

entonces la paciente llega a mí, recibe atención por mi parte, de ahí yo la derivo a la asistente social para hacer la respectiva visita, que se hace de forma integral, se atiende con psicóloga, durante un periodo de tiempo y si no se... como se dice.... se trata de que la paciente tenga herramientas psicológicas, herramientas que uno como médico pueda brindarle, aparte de los medicamentos, porque como te dije hay muchas con depresión. (MH, E3, L126)

De eso nos preocupamos, del hijo y de todo el hábitat de ellas, a veces no hay que enfocarse sólo en la paciente, sino en todo los que está alrededor de ellas y las causas que pueden producir esta violencia intrafamiliar. (MM, E2, L160)

Además en todos los perfiles se menciona como práctica la derivación a otras instancias de la red, específicamente a centros que se especializan en atender casos de este tipo, como también al segundo nivel de atención.

porque cuando son terapias más familiares, estamos derivando al asunto de familia que queda ahí en Barón. (PsM, E6, L34)

se deriva al centro de violencia intrafamiliar, que son acogidas en... por ejemplo el centro La Roca, también se derivan al centro de salud mental. (MH, E3, L132)

derivamos a alguna red social que generalmente tiene que ver con el centro de atención a mujeres que está en la municipalidad y se ingresa al programa de salud mental. (AS, E9, L33) 
Solo para algunos entrevistados resulta importante evaluar el riesgo vital, así como evaluar problemas a la salud mental de la mujer y de los hijos/as.

Evaluar el riesgo lo primero y qué tipo de violencia es, pero lo más importante es evaluar el riesgo, tomar alguna acción en violencia intrafamiliar y dependiendo del riesgo nosotros derivamos. (AS, E9, L31)

primero sintomatología, atacar los sintomas con los que viene: angustia depresión, ansiedad, crisis de pánico, lo que sea. (PsM, E5, L56)

que a veces la callan, se quedan calladitas y no se están dando cuenta que les están haciendo daño a los niños. (MM, E2, L163)

Respecto del tema de la judicialización de los casos, el tema de incentivar a las mujeres a realizar denuncias o constancias es planteada sólo por algunos entrevistados/as, de todos los perfiles.

Si vemos que es violencia física y si la paciente no ha puesto una constancia, la incentivamos a poner una, porque puede pasar a mayores, igual todo queda registrado y si tú eres la que atendiste... Ya pues, ¿y qué pasó doctora? (MM, E2, L31)

En este mismo tema resulta interesante la percepción que se tiene (psicólogo) de la posibilidad de recurrir a la ayuda institucional y judicial, la cual se aprecia como suficiente.

Esto es todo como un efecto dominó si produce eso a la vez produce el tema del temor, mujeres que no son capaces de, a pesar de que están todos los dispositivos puesto a disposición a nivel social, no son capaces de denunciar, eso me parece que es grave, por miedo. (PsH, E8, L155)

Por otro lado, para algunos/as entrevistados/ as era muy relevante intentar, en la medida que se pueda, trabajar en conjunto con la pareja la violencia contra la mujer al interior de esa relación, lo cual es visualizado como violencia cruzada, en el contexto de un conflicto conyugal.

Cuando yo veo que están las cosas que todavía se pude ayudar con consejerías, a lo mejor con técnicas de... para apoyarlos a ambos, porque no hay que darle la razón ni a uno ni al otro y a veces, ese también es otro error que se comete cuando unos le dan la razón a uno y al otro. (MM, E4, L298)

En general, en la mayoría de los discursos de los y las profesionales entrevistados, no hay evidencias de que éstos/as utilicen algo de las nociones o ideas que tienen sobre género y su relación con violencia contra las mujeres en el contexto de su relación de pareja en las intervenciones que realizan. Salvo en el caso de algunos/as psicólogos/as en que se puede ver una intencionalidad de utilizar confrontaciones en las ideas y estereotipos de género que presentan las consultantes. ahí el proceso es más largo, empezar a mirarse lo mal que se sienten emocionalmente, físicamente, lo disminuidas que se sienten lo poco atractivas, lo poco funcional, lo poco inteligente...eso, lo poco, lo poco que se sienten.... empezar a relacionar como lo ven a él y quien es él, quién es él, poh! Y por qué él puede hacer eso en ella, empezar a trabajar en el fondo todo lo que son y todo lo que no son ellas... lo que son al lado de él y lo que no son al lado de él, lo que son lejos de él y lo que no son lejos de él. Como hacer el paralelo y que lo que podrían y no hacer. (PsM, E5, L81)

Para un psicólogo en particular era de gran importancia poder realizar un trabajo de desvinculación de la imagen masculina con la de agresor, planteando a las usuarias otras posibilidades de modelos de masculinidad, lo cual evidentemente tiene una intencionalidad de trabajar el tema de las concepciones de género que tienen las usuarias.

para mí es muy importante desde la primera entrevista o cuando vienen mujeres violentadas que se atiendan conmigo, eso genera un cambio inmediato, yo siento que eso genera un cambio tremendamente positivo, porque tiene que ver con la figura de hombre, intentar comprender y hacerle entender a ella que esta figura que va a prestar una ayuda no tiene que ver con generalizar el género masculino frente al abuso. (PsH, E8, L146)

Un tema importante que surgió en general en todos los/as psicólogos/as y una asistente social, es el de potenciar a las mujeres en la toma de sus propias decisiones frente a la situación de violencia que enfrenta, lo cual está hablando de una intencionalidad de empoderar a las consultantes.

$Y$ principalmente son psicoterapias individuales para las mujeres en el fondo para reparar el daño y envalentonarlas para que hagan lo que tengan que hacer. (PsM, E6, L35)

Algunos entrevistados, consideran conveniente realizar en las intervenciones que practican sesiones donde se incluya a la mujer agredida junto con su pareja agresora, modalidad que no está recomendada en la literatura, ni en las guías clínicas que envía el MINSAL, e incluso para algunos autores es considerada negativa para el manejo de este tipo de problemas por la revictimización que ello puede generar en las mujeres ${ }^{22,23}$.

En cambio en la verbal siempre inyectar al paciente a que traiga a la pareja, porque qué hacemos usualmente tratamos a uno y el otro no quiere colaborar. Si vemos que el otro se hace reacio hacer una Interconsulta. (MM, E2, L34)

si yo ya siento que en la segunda entrevista tengo que citar al marido, lo cito(AS, E10, L179)

Primero que nada viene la mujer, es muy raro que venga la pareja, cuando uno ya dice que la 
señora traiga al esposo, porque siempre hay que escuchar las dos partes de la historia, entonces alli delante de él muchas veces las versiones cambian o a lo mejor tienen temor de decir lo que en realidad pasa, pero no es la misma historia que a uno le cuentan, después yo hago una sesión con la otra persona para saber su versión. (MM, E4, L33)

En el caso del perfil de médicos y médicas, ninguno menciona temas relacionados con intervenciones en donde exista la intención de plantear o evidenciar a las consultantes temas como: identificación de roles de género, identidad de género, empoderamiento, etc. lo que tampoco aparece en el grupo de asistentes sociales.

\section{Discusión y conclusiones}

La mayor disparidad en las percepciones detectadas en los discursos de los/as entrevistados/as se dio entre los diferentes tipos de profesionales y no entre el hecho de ser hombres o mujeres, que fueron los dos criterios de segmentación escogidos para determinar los perfiles de este estudio, lo cual difiere de los hallazgos de otras investigaciones ${ }^{11,14}$.

Dentro de este marco contextual, podemos encontrarnos con elementos positivos que se destacan de los discursos de los/as entrevistados/as esto es que existen ciertos consensos basales para todos/as ellos/as. En primer lugar es muy importante considerar que para ningún entrevistado es normal que exista violencia contra las mujeres en las relaciones de pareja, a todos/as los/as entrevistados/as les parece que es un problema relevante, todos consideran que hay que intervenir ante estas situaciones.

Hay consensos sobre la forma de abordar a las mujeres agredidas, para todos es muy relevante generar un espacio terapéutico acogedor, empático, de contención, etc. También hay mayor conocimiento sobre qué hacer en estos casos y existen nociones de la red a la cual acudir tanto desde el punto de vista de la asistencia sanitaria como desde el punto de vista del intersector. Todos estos elementos en la literatura revisada son reconocidos como importantes, pero no suelen ser considerados por los/as agentes sanitarios, según lo señalado en varias investigaciones ${ }^{9,15,16,21}$.

El hecho de que la mayor diferencia discursiva se haya dado entre las diferentes profesiones puede deberse a las diferentes formaciones que cada tipo de profesionales poseen, así aparece, a grosso modo, una mayor mirada biomédica de médicos y médicas, la visión psicosocial que está más presente en psicólogos, psicólogas y asisten- tes sociales lo cual resulta evidente dado las áreas en las que se forman unos/as y otros/as.

Sin embargo, respecto a la conceptualización general que existe en los discursos de médicos y médicas acerca de la violencia y las formas de abordaje de ésta, se puede apreciar una perspectiva menos biomédica que la esperable en los/as Médicos/as en general. Lo que se puede observar, por ejemplo en el abordaje integral y multidisciplinario del que se habla, de las derivaciones al intersector que se mencionan y sobretodo queda esa idea ya que se observa en sus discursos que no se esperan resoluciones inmediatas, exactas, uniformadas al problema que presentan las consultantes, en definitiva, se evidencia la intencionalidad de manejar estos casos de manera diferente a las consultas por otros problemas de salud.

Esto puede explicarse porque estos médicos y médicas tienen más tiempo dedicado a la salud mental lo que estaría hablando de una mayor experiencia en esas áreas, lo cual podría estar influenciando las maneras de plantearse frente a estos temas, algo similar a lo encontrado en otros estudios ${ }^{11}$.

Por otra parte, tal como lo señala la literatura revisada respecto del actuar de los agentes sani$\operatorname{tarios}^{12,13,15,17}$, aparecieron discursos en todos los perfiles, que presentaban algunos sesgos de género, los que llevaban a situaciones de invisibilización, revictimización, normalización y, en mayor o menor medida, a contradicciones dentro de la estructura discursiva.

Respecto de los sesgos de género que se observaron, se ve una forma de explicarse el fenómeno de la transmisión transgeneracional de formas relacionales violentas ligado a la figura materna, lo que de alguna forma es una manera de culpabilizar a las mujeres porque serían estas quienes estarían generando una sociabilización hacia la violencia.

Este fenómeno de la culpabilización de la figura femenina se observa también respecto de la violencia que se pueda generar contra las mismas mujeres. Esto lo podemos ver en planteamientos en donde se postula que las mujeres al no comprender a los hombres terminan hostigándolos o que las mujeres agreden psicológicamente a los hombres, a lo cual ellos responden con golpes o, tal vez, culparlas de la misma dependencia extrema que pudieran tener a sus parejas. Lo cual es coherente con el discurso hegemónico en tanto que deviene de mecanismos que contribuyen a perpetuar la violencia estructural hacia las mujeres ${ }^{27}$.

Hay situaciones en donde se evidencia invisibilización del problema de la violencia, de la mag- 
nitud o de los tipos de violencia, esto se ve, por ejemplo, cuando se describe una situación de violencia sexual la cual no es percibida como tal, sino atribuida a problemas de disfunción sexual en la mujer (falta de deseo sexual), lo cual desde nuestra perspectiva se trataría de violación. Esto evidencia una ausencia de discurso respecto de este tipo de violencia dentro de la pareja, lo cual, no es inhabitual, tanto en agentes sanitarios, como en las mismas mujeres agredidas sexualmente por sus parejas ${ }^{4}$, fenómeno que dice relación con creencias y actitudes arraigadas y que se relacionan con la noción de privilegios masculinos al interior de la relación de pareja, lo que es parte del discurso patriarcal hegemónico.

El mismo fenómeno se observa ante la ausencia de discurso frente a la violencia económica y el control masculino en la relación, el cual se invisibiliza a través de la figura de los celos, por ejemplo, elementos que en la literatura se han reconocido como situaciones de violencia de género en la pareja ${ }^{1,3}$.

El hecho de que algunas formas de expresión de la violencia sean invisibles para los/as funcionarios/as de salud, no es otra cosa más que el resultado de la invisibilización de estas a nivel de la sociedad entera y es, a su vez, un agente perpetuador y coproductor de invisibilidad.

Por otra parte el femicidio también aparece como poco visibilizado, probablemente esto se deba a que se trata de una situación que no tiene cercanía con el quehacer de la Atención Primaria, en donde se ven más bien las situaciones de violencia crónica y las consecuencias a largo plazo de la misma. Sumado, además, a la exposición que ha tenido el tema del femicidio en los medios de comunicación masiva, en donde se le ha dado un carácter de espectáculo en la prensa roja, lo cual lo aleja del ámbito cotidiano cada vez más. Resulta preocupante, la baja mención de la evaluación del riesgo vital de las usuarias que consultan por violencia.

Si bien en todos los relatos surgieron ciertas prácticas que llevan implícitas el evitar la revictimización como el acoger, escuchar, no juzgar ni criticar, cosas que se consideran así en la literatura y que mencionaron todos/as los/as entrevistados/ as como actitudes permanentes en ellos, también se mencionaron prácticas en donde hay mucho riesgo de generar revictimización esto, por ejemplo, durante la realización de sesiones en pareja.

Situaciones que al ser relatadas por los/as profesionales que las decían practicar eran percibidas, en ocasiones, como momentos de sufrimiento para las mujeres agredidas, lo cual se evi- denciaba por el temor que demostraban las mujeres en tales situaciones, pero que no eran experienciadas como situaciones de revictimización, que ellos/as mismos/as estaban generando.

En general, existe la percepción de que es un tema difícil de abordar debido -entre otras cosas- a que la violencia se encuentra normalizada y entonces se oculta o aparece encubierta en otros problemas de salud que llegan a la APS. Hay consenso respecto a que las mujeres agredidas no suelen consultar directamente por ello. Por lo cual se evidencia en los discursos sobre el abordaje de éstas, una valoración de temas como la actitud de sospecha y pesquisa permanente, ante indicios de violencia tales como molestias psicosomáticas, problemas de salud mental (estados ansiosos, depresivos, etc.) así como ante las consultas por problemas conductuales o emocionales de los /as hijos/as. La actitud de escucha en la acogida, la contención, la empatía son cualidades valoradas al enfrentar casos de maltrato, así como evitar los juicios de valor y críticas a las consultantes.

Se valora en los discursos el trabajo multidiciplinario que se hace en estos casos, siendo este más fuertemente expresado por médicos, médicas y asistentes sociales. También se plantea dentro de las intervenciones el manejo integral incorporando el discurso del modelo de Salud Familiar al análisis de los casos. Además, se refieren al uso de la red asistencial y de recurrir a las instancias de intersectorialidad que existen en la comuna, para el apoyo en el manejo de estos casos.

Hay concordancia en los discursos respecto de percibir dificultades en obtener como resultado de las intervenciones que se realizan el cese de la violencia, debido a la alta tasa de abandonos de tratamiento, las "recaídas", la dificultad y reticencia de las mujeres a poner fin a las situaciones de violencia, lo cual se acepta y se entiende como parte del problema, porque es visualizado como un elemento más que considerar en estos casos.

De las intervenciones que los y las profesionales entrevistados/as dicen realizar, en general, no existe en el discurso, una intencionalidad o direccionalidad hacia un empoderamiento desde una perspectiva de género, Salvo en el caso de algunos/as psicólogos/as.

Por todo esto, existe la percepción general de que no es fácil dar respuesta a este tipo de demanda. Por lo cual se aprecia cierta perplejidad ante la idea de estar presenciando situaciones de daño y desesperanza, en términos de que, a pesar de todas las cosas que se puedan realizar, los logros son bajos, entendidos éstos como la eli- 
minación de la violencia en la relación o la resolución de las consecuencias que ha acarreado el problema a la mujer y su familia. Aunque de todas formas se considera valorable la posibilidad de haber escuchado, acogido, apoyado a una mujer en situación de violencia ya que esto es percibido como un aporte para la posible solución del problema a largo plazo o simplemente como una posibilidad de válvula de escape que se les entrega a las consultantes.

\section{Referencias}

1. Organización Mundial de la Salud (OMS). La violencia en la Pareja. Informe Mundial sobre la violencia y la salud. Washington D.C.: OMS; 2003:95132.

2. Organizaçoes das Naciones Unidas (ONU). Declaración de de Naciones Unidas sobre la eliminación de la Violencia contra la Mujer. Washington, D.C.: ONU; 1993.

3. Organización de las Naciones Unidas (ONU), CEDAW. Comité para la Eliminación de la Discriminación contra la mujer, Recomendación general $N^{\circ} 19$. Washington, D.C.: ONU; 1992.

4. Asamblea General de Naciones Unidas (ONU). Resolución de la Asamblea General de Naciones Unidas. 58/147. Eliminación de la violencia contra la mujer en el hogar. In: Asamblea General de Naciones Unidas, AGdN, editor. Vol A/RES/58/147. Washington, D.C.: Asamblea General de Naciones Unidas; 2004.

5. Benhabib S, Cornell D. Teoría feminista y teoría crítica. Valencia: Alfons el Magnànim; 1990.

6. De Barbieri T. Sobre la categoría de género. Una Introducción teórico-metodológica. Debates en Sociología 1993; 18:145-169.

7. Garcia C, Cabral B. Socioantropología de la violencia de género. Revista de Estudios de Género 1999; 10:160-183.

8. Fernández S. Violencia de género en las prácticas institucionales de los programas de salud sexual y reproductiva como campo de estudio de la salud colectiva. IX Congreso Latinoamericano de Medicina Social. Lima: ALAMES; 2004.

9. Sugg NK, Thompson RS, Thompson DC, Maiuro R, Rivara FP. Domestic violence and primary care. Attitudes, practices, and beliefs. Arch Fam Med. 1999; 8(4):301-306.

10. Archer LA. Empowering women in a violent society. Role of the family physician. Can Fam Physician 1994; 40:974-983.

11. Eguiguren P, García-Calvente MM. Percepciones, conocimientos, actitudes y prácticas de médicos y médicas Atención Primaria sobre Violencia de género. Granada: Escuela Andaluza de Salud Pública; 2002.

12. Rodriguez MA, Bauer HM, McLoughlin E, Grumbach K. Screening and intervention for intimate partner abuse: practices and attitudes of primary care physicians. JAMA 1999; 282(5):468-474.

13. McLeer SV, Anwar R. A study of battered women presenting in an emergency department. Am J Public Health 1989; 79(1):65-66.

14. Lo Fo Wong SH, De Jonge A, Wester F, Mol SS, Romkens RR, Lagro-Janssen T. Discussing partner abuse: does doctor's gender really matter? Fam Pract. Oct 2006; 23(5):578-586.

15. Corsi J. ¿Cómo se puede prevenir la violencia en la pareja? In: Echeburua E, De Corral P, editores. Manual de Violencia Familiar. Madrid: Siglo XXI de España Editores; 1999. p.177-188.

16. Rhodes KV, Levinson W. Interventions for intimate partner violence against women: clinical applications. JAMA 2003; 289(5):601-605.

17. Ramos L. Reflexiones para la comprensión de la salud mental de la mujer maltratada por su pareja íntima. La Ventana 2002; 16:130-181. 
18. Méndez CL. Violencia en la pareja. In: Maturana $\mathrm{H}$ CF, Montenegro H, Kunstmann G, Méndez C, editores. Violencia en sus distintos ámbitos de expresión. Santiago: Dolmen Ediciones; 1995. p. 23-38.

19. Casanova V, Suit S. Diagnóstico de personal de salud y usuarias sobre violencia intrafamiliar y la atención del problema por parte de salud. Santiago: Ministerio de Salud, Chile (MINSAL); 1996.

20. Chile. Ministerio de Salud (MINSAL). Prioridad Programática: trastornos de la salud mental asociados a la violencia Mujeres y adultos mayores afectados por violencia intrafamiliar. Santiago: MINSAL; 1997.

21. Chile. Ministerio de Salud (MINSAL). Manual de apoyo técnico para las acciones de Salud en violencia intrafamiliar. Santiago: MINSAL; 1998.

22. Chile. Ministerio de Salud (MINSAL). Guía Clínica para la Atención Primaria: Violencia Intrafamiliar, Detección Diagnóstico y Tratamiento. Santiago: MINSAL; 2004.

23. Chile. Ministerio de Salud (MINSAL). Manual para la atención primaria: Intervención Psicosocial de grupo para el tratamiento de la violencia intrafamiliar. Santiago: MINSAL; 2004.

24. Organización Panamericana de Salud (OPAS). Violencia de género: gran problema, insuficientes respuestas. In: Organización Panamericana de Salud (OPAS). Observatorio de equidad de género en salud: informe 2005. Santiago: OPAS; 2006. p. 33-45.

25. Souza M. El desafío del conocimiento, Investigación cualitativa en salud. Buenos Aires: Editorial Lugar; 2004.

26. Berger PLT. La construcción social de la realidad. Madrid: Amorrortu-Murguia; 1984.

27. Lorente M. Mi marido me pega lo normal agresión a la mujer: realidades y mitos. Barcelona: Ares y Mares Bolsillo; 2003.

Artigo apresentado em 10/07/2011

Aprovado em 15/08/2011

Versão final apresentada em 03/10/2012 